\title{
Disparities in Telehealth for Liver Disease: Revealing What Was Hidden in Plain Sight
}

\author{
Nabeel Wahid ${ }^{1} \cdot$ Russell Rosenblatt $^{1}$
}

Accepted: 7 April 2021 / Published online: 4 May 2021

(c) The Author(s), under exclusive licence to Springer Science+Business Media, LLC, part of Springer Nature 2021

Disparities in liver disease care, including life-saving liver transplantation, have been well documented across socioeconomic status, race, gender, age, and geography [1]. Unfortunately, the COVID-19 pandemic, by disproportionately affecting vulnerable groups, has exacerbated pre-existing inequities in healthcare. In the realm of liver disease, the impact of the COVID-19 pandemic on social determinants of health in liver disease has touched all aspects of care. This is exemplified by reduced access to substance abuse therapy for patients with alcohol use disorder, food insecurity in patients with nonalcoholic fatty liver disease, and limited access for all patients to appropriate screening tests.

One of the few positive developments of the pandemic has been a change in healthcare delivery due to the rapid rise of telehealth. While telehealth utilization in hepatology prior to the pandemic was quite uncommon and mostly limited to initiatives aimed at increasing access for Veterans and for those living in rural areas [2], increased reimbursements for telehealth visits and the need to continue safely caring for liver patients during the pandemic substantially increased utilization. In a survey of liver and intestinal transplant programs, only $16 \%$ of institutions used telehealth prior to the pandemic but $98 \%$ were utilizing telehealth by April 2020 [3].

It is in this environment that Wegermann et al. [4] performed an important analysis of disparities in telehealth use for liver diseases, reported in this issue of Digestive Diseases and Sciences. The authors retrospectively compared completed telehealth video visits with telephone calls and incomplete visits both before and during the pandemic at a large academic health system. This study was designed to reveal an important finding that was right in front of our faces-namely, that despite the increase in telehealth

Russell Rosenblatt

rur9017@med.cornell.edu

1 Department of Medicine, Weill Cornell Medicine, 1305

York Avenue, 4th floor, New York, NY 10021, USA providing improved access for some, vulnerable patients are being left behind.

The authors noted that non-Hispanic Black patients, public insurance, older age, and single marital status were all associated with an increased risk of completing a telephone visit instead of a video visit. These findings, though of importance, are unfortunately unsurprising given that these groups face disparities in many other aspects of care. The authors used a multinomial regression model controlling for covariates that were selected a priori, further bolstering the credibility of their association. Moreover, these findings are echoed by two studies in different regions of the USA-an otolaryngology clinic in Detroit [5] and a gastroenterology clinic in Philadelphia [6].

The findings in this study demonstrate a disparity in access to care. Though the inherent assumption is the authors' assertion that video visits are superior to telephone visits, does this outcome truly measure an impactful disparity? Video visits have implicit benefits-including but not limited to the ability to detect physical findings such as sarcopenia, ascites, jaundice, and asterixis. Furthermore, it can be helpful to see a patient in order to form a face-to-face connection. That being said, since effective video telehealth utilization is not a part of most formal training programs in hepatology, there is likely to be considerable heterogeneity in effectiveness of video visits among providers.

Telephone visits do provide adequate information for most patients, particularly those who are less acutely ill or with whom there is an established relationship. A study performed by Britton et al. in the UK focusing on patients with chronic gastrointestinal conditions found that patients were quite satisfied with telephone calls; the addition of video visits did not substantially improve satisfaction [7]. In contrast, Serper et al. in the USA assessed telehealth satisfaction and usage early in the pandemic, finding that while nearly two-thirds of patients are satisfied with a telephone visit, only $41 \%$ of physicians are satisfied, markedly less than the $88 \%$ satisfied with video visits [6]. Despite these conflicting 


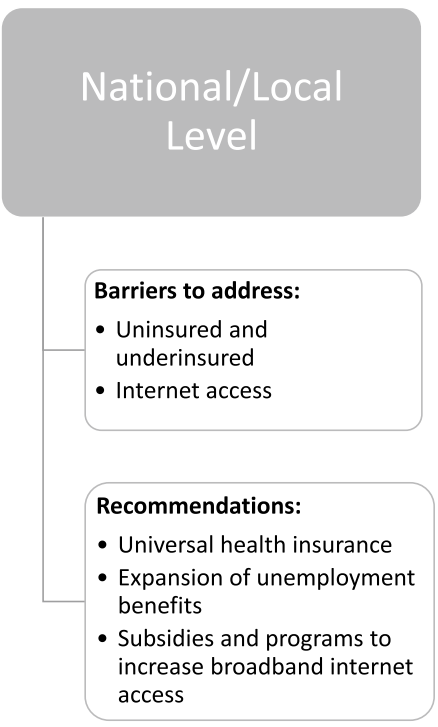

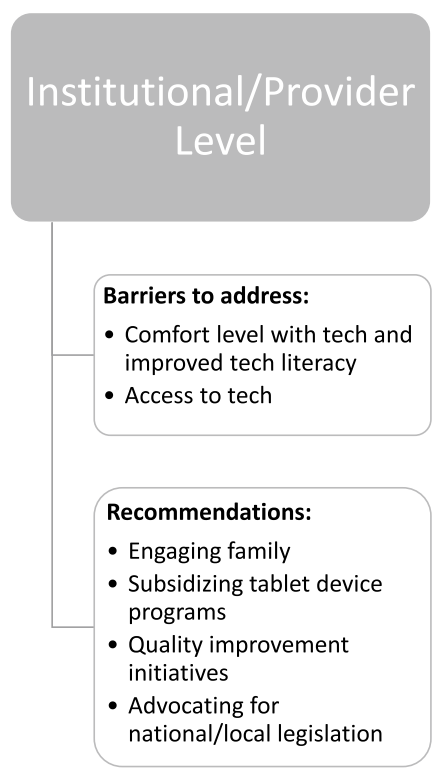

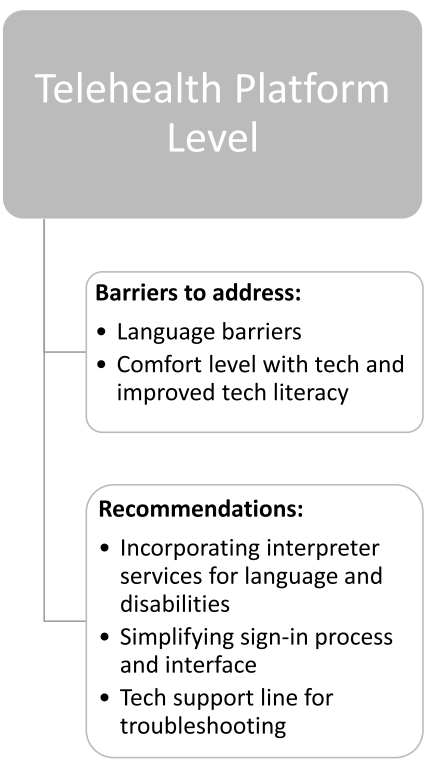

Fig. 1 Multi-level approach to reduce barriers to telehealth disparitiesCaption: The above figure includes the three levels where disparities can form and recommendations to overcoming these barriers

findings, given that the video visit was the preferred visit method and specific populations used it less, we do agree with the authors that these findings demonstrate a true disparity that matters. Still, it is unclear whether this disparity impacts liver-related outcomes or is rather a surrogate marker for poor access to care in general.

Future analyses would benefit from more detailed socioeconomic data to facilitate further targeting of who is at a disadvantage. Insurance, as utilized in this analysis, has been used in countless studies as a surrogate marker of socioeconomic status but has its limitations, especially in a pandemic in which tens of millions lost their jobs and became food insecure. Currently, assessing socioeconomic status is a moving target. Furthermore, with time and more experience in telehealth, narrower geographic categories may provide newer insights. Although distance from a large academic liver transplant center is certainly important in predicting future need, use, and access to telehealth, there may be substantial heterogeneity. The use of county-level data with other socioeconomic measurements, such as social vulnerability index, can help determine the susceptibility to disparate outcomes of patients. Acknowledging these limitations, the geographic group that would likely benefit most from continued telehealth management, patients living greater than 50 miles from the clinic, were more likely to complete video visits.

Prior literature has noted well-documented geographic disparities in liver disease care, including access to liver transplant between urban and rural communities [8]. Although this study did not assess disparities in telehealth based on population density, many of the telehealth initiatives prior to the pandemic were targeted toward rural communities with limited access to subspecialists. One could theorize that the existing infrastructure and reliance on telehealth in rural communities prior to the pandemic would have been an asset during the pandemic. Nevertheless, at the same time, limited access to high-speed Internet in rural communities disadvantages these patients who are remote from major academic centers. Focusing on improving visit quality in patients living in rural communities far from hepatology services is essential.

To effectively chart a path forward, it is necessary to move past what was considered acceptable in times of rapid adoption, lockdown, and crisis. During the height of the COVID-19 pandemic, a reasonable approach seemed to be that the best telehealth modality to use is the one that connects a patient with their provider. If this access is by telephone call, it is still an improvement over total lack of medical care in times of shutdown. Nonetheless, planning for the routine implementation of telehealth nationwide requires higher expectations. Healthcare providers cannot simply accept an incremental improvement that leaves the same vulnerable patients behind.

Some of the solutions require national and local legislation to improve our patients' access to care (Fig. 1). Too many patients remain uninsured, before even accounting for the loss of tens of millions of jobs and not including highdeductible healthcare plans that can disincentivize patients from seeking care or ration care. Improving broadband access, especially in rural areas, needs to be a national priority. Healthcare providers need to vocally advocate for our patients without a voice and who are suffering from inequity. 
Still, smaller and simpler solutions, which need to be implemented today, can help narrow the disparity gap. We have to make patient portals easy to access with the goal of minimization or avoidance of potential roadblocks in connection to care. One such example is to avoid the requirement of smart telephone applications and instead provide Web site-accessible platforms. Ensuring that telehealth platforms are available in a multitude of languages and facilitating easy access to an interpreter services, including American Sign Language, are essential to keep all patients on equal footing. Also, elderly patients with lower comfort levels with telehealth technology may benefit from dedicated support lines in order to help troubleshoot issues and test video connections prior to video visits. Moreover, providers should engage family, especially children or grandchildren, to help manage technology that may be challenging for older patients. Healthcare institutions could help subsidize the purchase of tablet device programs in order to ensure that those without appropriate hardware for telehealth at home can still have access. Finally, now that disparities in telehealth have clearly been identified, researchers should focus on quality improvement initiates to promote equity in telehealth at the departmental and institutional level.

The COVID-19 pandemic forced the adoption of telehealth and its use will likely never decline to pre-pandemic levels. The rapid expansion of telehealth during the COVID19 pandemic is an accomplishment in its own right. Nonetheless, like most new technologies, there exists an ability to ameliorate or exacerbate disparities. This important work highlights that the same vulnerable patients who suffer from healthcare disparities and outcomes are also at a disadvantage in access to optimal telehealth access. The next steps must focus on expanding the reach of telehealth and ensuring that we do not leave vulnerable groups behind.

\section{References}

1. Wahid N, Rosenblatt R, Brown RS Jr. A review of the current state of liver transplantation disparities. Liver Transplant. 2020. https:// doi.org/10.1002/lt.25964.

2. Su GL, Glass L, Tapper EB, Van T, Waljee AK, Sales AE. Virtual consultations through the veterans administration SCAN-ECHO project improves survival for veterans with liver disease. Hepatology. 2018. https://doi.org/10.1002/hep.30074.

3. Sherman CB, Said A, Kriss M, Potluri VS, Levitsky J, Reese PP et al. In-Person outreach and telemedicine in liver and intestinal transplant: a survey of national practices, impact of coronavirus disease 2019, and areas of opportunity. Liver Transplant. 2020. https://doi.org/10.1002/lt.25868.

4. Wegermann K, Wilder J, Parish A, Niedzwiecki D, Gellad Z, Muir A, et al. Racial and Socioeconomic Disparities in Utilization of Telehealth in Patients with Liver Disease During COVID19. Dig Dis Sci. (Epub ahead of print.) https://doi.org/10.1007/ s10620-021-06842-5.

5. Darrat I, Tam S, Boulis M, Williams AM. Socioeconomic disparities in patient use of telehealth during the coronavirus disease 2019 Surge. JAMA Otolaryngol Head Neck Surg. 2020. https:// doi.org/10.1001/jamaoto.2020.5161.

6. Serper M, Nunes F, Ahmad N, Roberts D, Metz DC, Mehta SJ. Positive early patient and clinician experience with telemedicine in an academic gastroenterology practice during the COVID-19 Pandemic. Gastroenterology. 2020. https://doi.org/10.1053/j.gastro.2020.06.034.

7. Britton E, Richardson P, Mian I, Conley T, Byrne D, Boyd H et al. Letter: Covid-19-re-initiating clinical services for chronic gastrointestinal diseases. How and when? Aliment Pharmacol Ther. 2020;2:1. https://doi.org/10.1111/apt.16061.

8. Goldberg DS, French B, Forde KA, Groeneveld PW, Bittermann $\mathrm{T}$, Backus L et al. Association of distance from a transplant center with access to waitlist placement, receipt of liver transplantation, and survival among US Veterans. JAMA J Am Med Assoc. 2014. https://doi.org/10.1001/jama.2014.2520.

Publisher's Note Springer Nature remains neutral with regard to jurisdictional claims in published maps and institutional affiliations. 